\title{
Valoración de un proyecto de "triage" de urgencias por enfermería en atención primaria
}

\author{
María Candelaria Ayuso Rayaa ${ }^{a}$ Nieves Pérez López ${ }^{\mathrm{b}}$, María José Simarro Herráez ${ }^{\mathrm{b}}$, \\ y Francisco Escobar Rabadána
}

\begin{abstract}
a Médico de Familia. Centro de Salud Universitario Zona-IV. Albacete (España)

${ }^{b}$ Diplomada Universitaria en Enfermería. Centro de Salud Universitario Zona-IV. Albacete (España)
\end{abstract}

Correspondencia: María Candelaria Ayuso Raya. Centro de Salud Universitario Zona-IV. C/ Seminario, 4. C.P. 02006. Albacete (España). Correo electrónico: candeayuso@ hotmail.com

Una versión preliminar de este trabajo fue presentada en el XII Congreso de Atención Primaria de Castilla-La Mancha:

"Atención Primaria: la Primera Atención", celebrado en

Cuenca los días 12,13 y 14 de Mayo de 2011.

La versión definitiva del mismo fue presentada en las I Jornadas de Investigación de Revista Clínica de Medicina de Familia, celebradas en Ciudad Real los días 21 y 22 de junio de 2013.

Recibido el 19 de septiembre de 2013.

Aceptado para su publicación el 12 de octubre de 2013.

\begin{abstract}
RESUMEN
Objetivo: Conocer la valoración, por parte de enfermeras y estudiantes de enfermería, sobre cambios organizativos para la instauración de un sistema de "triage" de urgencias en atención primaria.

Diseño del estudio: Estudio cualitativo, tipo grupo focal.

Emplazamiento: Atención primaria.

Participantes: Enfermeras de atención primaria y estudiantes de tercer curso de enfermería.

Método: Se constituyeron dos grupos, uno formado por siete enfermeras, pertenecientes a centros de salud urbano, rurales y SUAP; y un segundo grupo formado por cinco estudiantes de tercer curso de enfermería. Se realizó una videograbación de las reuniones y posteriormente se llevó a cabo un proceso de análisis y síntesis de la información.

Resultados: Se constató un acuerdo en ambos grupos sobre la factibilidad de esta tarea y, en general, se consideraban con suficiente cualificación para su desempeño. Se subrayó asimismo la importancia de que no recaiga sobre el área administrativa la responsabilidad de decidir quién y cuándo debe atender a un paciente que requiere atención inmediata. En el grupo de enfermeras se hizo especial hincapié en que este proyecto no sería viable con las cargas de trabajo actuales, por lo que planteaban la necesidad de aumentar las plantillas o introducir cambios organizativos. Los estudiantes se mostraban interesados por asumir este tipo de tareas, aunque desearían una mayor formación específica en su currículo académico. Consideraban fundamental delimitar tareas y protocolizar actividades.

Conclusiones: Enfermeras cualificadas en el trabajo en atención primaria y estudiantes de enfermería en la fase final de su formación coinciden en la factibilidad de implantar un sistema de "triage" para pacientes que requieren atención inmediata en un centro de salud.
\end{abstract}

PALABRAS CLAVE: Triaje, Medicina de Emergencia, Enfermería de Atención Primaria.

\section{ABSTRACT}

Evaluation of a primary care nursing emergencies triage project.

Objective: To ascertain the evaluation of nurses and nursing students on organisational changes for the establishment of a triage system for primary care emergencies.

Design: Focus group qualitative study.

Location: Primary Care.

Participants: Primary health care nurses and third year nursing students.

Method: Two groups were formed, one of seven nurses from city, rural and SUAP healthcare centres and a second group made up of five third year nursing students. A video recording was made of the meetings and later a process of analysis and synthesis of the information was carried out.

Results: There was agreement between both groups on the feasibility of this task and, in general, they considered themselves sufficiently qualified to perform it. Likewise, importance was given to the fact that responsibility for deciding who should attend to a patient needing immediate care, and when, should not fall on the administrative area. In the nursing group, special emphasis was placed on the fact that this project would not be viable with current workloads and, for this reason, they put forward the need to increase the workforce or to bring in organisational changes. The students showed interest in taking on this kind of task, although they would wish for greater specific training in their academic curriculum. They considered definition of tasks and regulation of activities to be fundamental.

Conclusions: Qualified nurses at work in primary care and nursing students nearing the end of their training agree on the feasibility of implementing a triage system for patients requiring immediate attention at a healthcare centre. 


\section{INTRODUCCIÓN}

La palabra "triage" procede de un vocablo francés (trier) que quiere decir "elegir o clasificar", y se ha aceptado para la acción de clasificación de enfermos. El primer profesional de la salud en utilizar el término "triar" fue el barón DominiqueJean Larrea (1766-1842), médico cirujano militar. En las guerras napoleónicas creó el transporte en ambulancia e introdujo los principios de la sanidad militar moderna, realizando los primeros "triages" como un sistema de clasificación para tratar a los heridos en el campo de batalla.

En el ámbito hospitalario de las urgencias, el "triage" se introdujo a principios de los años sesenta en Estados Unidos, debido al aumento de la población que diariamente acudía a dichos servicios con un elevado porcentaje de patologías no urgentes, desarrollando un sistema clásico de clasificación en tres niveles de categorización, que fue sustituido a finales del siglo pasado por un nuevo sistema de cuatro niveles. Estos sistemas no han conseguido un grado de evidencia científica suficiente como para ser considerados estándares del "triage" moderno".

Paralelamente al nacimiento del sistema americano, en Australia, en el año 1933, se fue consolidando la National Triage Scale for Autralasian Emergency Departments (NTS), siendo la primera escala con ambición de universalización basada en 5 niveles de priorización. En el año 2000, la NTS fue revisada y recomendada como Escala australiana de "triage" (Australasian Triage Scale: "ATS"). Tras su implantación, en diferentes países, se han ido desarrollando modelos de "triage" que han adaptado sus características, y que han tenido como objetivo la implantación de un sistema de "triage" de urgencias universal, en sus respectivos territorios.

Actualmente existen cinco sistemas, escalas o modelos de "triage" que, además del australiano, incluyen: la Escala canadiense de "triage" y urgencia (Canadian Emergency Department Triage and Acuity Scale: "CTAS"), el Sistema de "triage" de Manchester (Manchester Triage System: "MTS"), el Índice de severidad de urgencias de 5 niveles de "triage" (Emergency Severity Index 5 level triage: "ESI"), desarrollado por el Grupo de trabajo del ESI en los EE.UU. en 1999, y el Modelo andorrano de "triage" (Model Andorra de triatge: "MAT")2.

Un buen sistema de "triage" debe cumplir con las propiedades de reproducibilidad, utilidad y validez, lo que implica tener una buena concordancia interobservador, ser útil para medir el grado de urgencia, tener validez empírica para predecir la gravedad y la evolución de los pacientes, ser aplicable a cualquier servicio de urgencias,

1. Clasificar los pacientes según la gravedad y la severidad de los signos y síntomas e identificar rápidamente los enfermos con riesgo vital inminente.

2. Ordenar de manera eficaz la demanda mediante la valoración enfermera inicial.

3. Mejorar la calidad y la eficacia de los servicios de urgencias y emergencias mediante una respuesta adecuada a las necesidades de los usuarios, estableciendo criterios homogéneos, científicos y coherentes acerca de la necesidad de atención que el paciente presenta con respecto a su demanda de asistencia y, según esto, aplicar las intervenciones enfermeras para garantizar su seguridad.

4. Determinar el área de tratamiento adecuada, priorizando la asistencia cuando la situación del servicio origina una espera para la visita.

5. Iniciar pautas terapéuticas iniciales protocolizadas.

6. Disminuir la congestión de las áreas de tratamiento y determinar el área más adecuada para tratar un paciente que se presenta en el servicio de urgencias.

7. Garantizar que las necesidades de atención del paciente son reevaluadas durante su espera. Informar a los pacientes y familiares acerca de la asistencia y el tiempo aproximado de espera.

8. Orientar a los pacientes a otros niveles de asistencia de la institución si lo requieren.

9. Actuar de interlocutor con los miembros de los equipos de transporte sanitario.

10. Proporcionar información acerca del proceso de acogida, clasificación y demoras en la atención médica que permita analizar y mejorar la gestión del servicio.

11. Prestar a los pacientes y a sus familiares o acompañantes, una aproximación humana y profesional al problema planteado como demanda asistencial en todo su contexto.

Tabla 1. Objetivos que deberían cumplirse en la realización del "triage" por parte de enfermería (Puigblanqué et al.') 
ser aplicable a adultos y niños y disponer de indicadores de calidad propios de la escala. En la tabla 1 se recogen los objetivos que, siguiendo a Puigblanqué et $\mathrm{al}^{1}$, deberían cumplirse en la realización del "triage" por parte de enfermería.

En nuestro país, la atención urgente se lleva a cabo principalmente desde 3 niveles asistenciales: Servicios de urgencias hospitalarios, Servicios de emergencias extrahospitalarios y Servicios de urgencias de atención primaria (SUAP) ${ }^{3}$.

Disponemos de un estándar de "triage" estructurado propio, el Sistema Español de "Triage" (MAT-SET), adaptación del MAT realizada en el año 2003 por la Sociedad Española de Medicina de Urgencias y Emergencias (SEMES) ${ }^{2}$. Ha demostrado su aplicabilidad y su capacidad de adaptación y mejora continua gracias a una amplia experiencia de implantación. En la tabla 2 se recogen los niveles de urgencia que establece.

Que el "triage" estructurado es un proceso crítico para el manejo eficiente y eficaz de los servicios de urgencias modernos ya no forma parte del catálogo de cuestiones discutibles en medicina y enfermería de urgencias y emergencias; sin embargo, en los organizativos para valorar la instauración de un proyecto piloto sobre el sistema de "triage" de urgencias en atención primaria.

\section{SUJETOS Y MÉTODOS}

Se trata de un estudio cualitativo, tipo grupo focal, realizado en el mes de abril de 2010 en la sala de reuniones del Centro de Salud Zona IV de Albacete y en el que se incluyeron enfermeras de atención primaria y estudiantes de tercer curso de enfermería.

Se constituyeron dos grupos focales: uno formado por siete enfermeras con amplia experiencia profesional, con edades comprendidas entre los 40 y los 55 años y pertenecientes a centros de salud urbano, rurales y SUAP; y un segundo grupo formado por cinco estudiantes, cuatro mujeres y un hombre, de tercer curso de enfermería. En cuanto a las enfermeras cualificadas, se realizó un proceso de selección de profesionales de diferentes ámbitos de la atención primaria, a través de contacto telefónico o personalmente, quienes decidían su participación en función de su interés personal. Todas conocían cómo funciona un sistema de "triage", y alguna de ellas (enfermera

- $\quad$ Nivel I (Resucitación): Situaciones que requieren resucitación, con riesgo vital inmediato.

- $\quad$ Nivel II (Emergencia): Situaciones de emergencia o muy urgentes, de riesgo vital previsible, la resolución de las cuales depende radicalmente del tiempo.

- $\quad$ Nivel III (Urgencia): Situaciones de urgencia, de potencial riesgo vital.

- $\quad$ Nivel IV (Menos urgente): Situaciones de menor urgencia, potencialmente complejas, pero sin riesgo vital potencial.

- $\quad$ Nivel $\mathbf{V}$ (No urgente): Situaciones no urgentes, que permiten una demora en la atención o pueden ser programadas, sin riesgo para el paciente.

Tabla 2. Niveles de urgencia según el Sistema Español de "Triage" (MAT-SET)

servicios de urgencias de atención primaria este proceso está siendo más lento, en parte por no existir herramientas propias (debemos considerar la mayor incidencia en este nivel de patología menor, debiendo ser más sensibles a patologías más graves, por lo que no serían extrapolables los modelos clásicos del "triage") y tener que utilizar sistemas creados para los servicios hospitalarios, suponiendo su implantación un importante desembolso económico ${ }^{2,3}$.

El objetivo de nuestro estudio es conocer la valoración, por parte de enfermeras y estudiantes de enfermería, de una propuesta sobre cambios del SUAP) tenía experiencia sobre su aplicación en las urgencias de atención primaria.

El diseño del proyecto de valoración elaborado a tal efecto fue entregado previamente a los participantes por los investigadores principales a modo de hoja informativa. Estaba organizado en dos bloques, uno con relación a los objetivos de la propuesta organizativa y un segundo bloque en relación con las medidas propuestas para su implantación en el centro. Entre los objetivos propuestos para el debate se plantearon: la agilización de la asistencia a los pacientes que requieren atención inmediata, diferenciando aquellas demandas que pueden 
ser perfectamente diferibles, lo que supone una descarga de responsabilidad a los auxiliares administrativos en la gestión de las demandas urgentes, evitando que los pacientes tengan que explicar sus motivos de consulta en el área administrativa, con la posible violación del derecho a la intimidad, haciendo efectivo el objetivo, tanto institucional como profesional, de que enfermería atienda la consulta de demanda, medida que en consecuencia evitaría presumiblemente las interrupciones de la actividad asistencial de los profesionales del centro de salud. En cuanto a las medidas propuestas a ambos grupos, planteamos que aquellos pacientes que soliciten asistencia en el centro de salud deberán comunicarlo inexcusablemente en el área administrativa, ya sea de forma presencial o por teléfono. Las agendas médicas incluirán huecos de cita para "urgencias" (deberíamos hablar más bien de "huecos para pacientes sin cita previa") todos los días. Esto permitirá diferenciar agendas de "demanda demorable" (para los que se debería admitir la existencia de "listas de espera") y "demanda no demorable" (visita en el día).

Se realizó una videograbación de ambos grupos, previo consentimiento informado de los participantes, y posteriormente se llevó a cabo un proceso de análisis de las opiniones y síntesis de la información recogida agrupando las opiniones de enfermeras y estudiantes bajo epígrafes, lo que nos ha permitido homogenizar criterios y llegar a un análisis más preciso sobre la valoración del proyecto.

\section{RESULTADOS}

Tras la revisión pormenorizada de las opiniones de ambos grupos (enfermeras y estudiantes de tercer año), constatamos un acuerdo general sobre la factibilidad en la realización de un proyecto de "triage". En las tablas 3 y 4 presentamos las opiniones encuadradas en los epígrafes que consideramos de mayor relevancia para ambos grupos.

En el grupo de enfermeras se hizo especial hincapié en que este proyecto no sería viable con las cargas de trabajo actuales. Proponían la necesidad de aumentar las plantillas o bien la introducción de cambios organizativos que permitieran redistribuir tareas que pudieran ser asumibles por otros profesionales, subrayando asimismo la importancia de que no recaiga sobre el área administrativa la responsabilidad de decidir quién y cuándo debe atender a un paciente que requiere atención inmediata, considerando prioritario como medida de gestión adicional la necesidad de disponer de métodos de trabajo objetivos, cuantificables y fácilmente reproducibles que permitan medir la magnitud de las demandas, la optimización de recursos y sobre todo aquellos susceptibles de ser modificados.

Se manifestaron con firmeza ante la premisa en cuanto a que la decisión de si es o no demorable nunca será del paciente, pero tampoco del auxiliar administrativo. Éste se limitará a dar cita al paciente con uno u otro profesional según la preferencia (en cuanto a demora admitida de asistencia) del paciente. La enfermera de guardia recibirá estas llamadas, o visitará (si el paciente ya se encuentra en el centro de salud) en su misma consulta, y les dará respuesta automática: ser valorado por ella misma en la consulta, cita en la agenda de "visita en el día" de su médico, cita con el "médico de guardia" si el médico del paciente no está presente en el centro de salud, o cita en la "agenda de demanda demorable" de su médico. Incluso podría tomar decisiones sobre su adecuación de visita a domicilio, o pasar a otros servicios asistenciales como 112, SUAP o derivación a urgencias hospitalarias.

Los estudiantes se mostraban interesados por asumir este tipo de tareas, aunque desearían una mayor formación específica en su currículo académico.

Pensaban que la implantación de este modelo organizativo podría chocar inicialmente con cierta resistencia al cambio, y que sería fundamental delimitar tareas y protocolizar actividades como escala de priorización útil y fácilmente reproducible para relacionar la asignación adecuada del paciente.

\section{DISCUSIÓN}

La Sociedad Española de Enfermería de Urgencias asume y propone que la actividad de recepción, acogida y clasificación "triage" sea realizada por enfermeros, siendo una actividad propia de enfermería, como así lo reconocen los artículos 52, 53 y 54 del Real Decreto 1231/2001, de 8 de noviembre y la Ley $44 / 2003$, de 21 de noviembre, de ordenación de las profesiones sanitarias en sus artículos 5.1 , a) b) y c); artículo 7.1 y 2 a).

En un servicio de urgencias con un sistema de "triage" se pretende establecer una dinámica de grupo, de manera que los profesionales de enfermería por su cualificación profesional sean los 


\begin{tabular}{|c|c|}
\hline Valoración inicial & $\begin{array}{l}\text { "Si, es posible, con una distribución distinta del trabajo". } \\
\text { "Necesitamos más profesionales, tenemos que ser conscientes de lo que no tenemos". } \\
\text { "Posible mediante una distribución distinta de la consulta y las tareas de enfermería". } \\
\text { "Somos los profesionales más adecuados". } \\
\text { "Habrá situaciones demorables, no es una tarea fácil". } \\
\text { "Aceptamos cambios organizativos, aunque la enfermería tiene sus tiempos". } \\
\text { "Conoces perfectamente tu sistema de trabajo, aceptamos la urgencia como puerta de } \\
\text { entrada". } \\
\text { "No debe ser el administrativo el que realice la tarea de "triage". } \\
\text { "Con los objetivos estoy totalmente de acuerdo, aunque tengo mis dudas sobre si enfermería } \\
\text { es capaz de resolver el 100\%". } \\
\text { "La enfermería está en condiciones de actuar". } \\
\text { "No tengo que diagnosticarlo" "Es necesario priorizar lo urgente". } \\
\text { "Esto que proponéis me encanta". }\end{array}$ \\
\hline Punto de partida & $\begin{array}{l}\text { "Señalamos que las enfermeras desde el año } 2008 \text { atendemos diariamente la demanda } \\
\text { así como la consulta programada. Atiende la demanda en su puerta y la prioriza, valora la } \\
\text { adherencia a los tratamientos, realiza curas locales, información sobre la prescripción". } \\
\text { "Estamos de acuerdo en cambios en el punto de entrada del paciente. Necesaria la vehiculación } \\
\text { de un paciente que hoy por hoy es el médico el que recepciona inicialmente". } \\
\text { "Los puntos de vista son distintos dependiendo si trabajas en un centro rural, en un centro } \\
\text { urbano o en un SUAP". } \\
\text { "Si eres una enfermera única tienes que hacer "triage", curar, atender, dar educación sanitaria, } \\
\text { y todo eso forma parte de nuestro trabajo, pero tiene sus tiempos". } \\
\text { "Existe un problema de tiempos. Existe un problema de personal. Hablamos de redistribuir } \\
\text { personal". } \\
\text { "Se debe evitar el motivo de consulta en el mostrador". } \\
\text { "En el pueblo el cribaje lo hace el mostrador, es incluso amenazante para derivar el paciente al } \\
\text { primer enfermero que circula". }\end{array}$ \\
\hline Cómo organizar & $\begin{array}{l}\text { "Crear la figura de enfermera de incidencias ya existente en centros rurales". } \\
\text { "Establecer un rotatorio definido para gestión de tiempos". } \\
\text { "Necesaria la propia gestión de la agenda diaria". } \\
\text { "Proceso de implantación depende del volumen de población". } \\
\text { "Es un momento para sentarnos, ver agendas, planificar". } \\
\text { "Atendiendo las urgencias que lleguen al centro de salud telefónicamente y presencialmente". } \\
\text { "Aceptamos el proyecto". } \\
\text { "Donde enfermería ha hecho "triage" ha habido un gran porcentaje de éxitos, alto grado de } \\
\text { satisfacción en pacientes y familiares". } \\
\text { "La enfermera debe saber priorizar". } \\
\text { "Desde la Gerencia se deben plantear cambios organizativos sin que suponga un enfrentamiento } \\
\text { "La consonal con el equipo". } \\
\text { Necesitamos informatizarlo, registrarlo". }\end{array}$ \\
\hline $\begin{array}{l}\text { Necesidades } \\
\text { formativas }\end{array}$ & $\begin{array}{l}\text { "La enfermera de "triage" se debería formar". } \\
\text { "La labor de "triage" requiere formación y puesta a punto, es muy importante. No forma parte } \\
\text { de nuestra labor diaria". }\end{array}$ \\
\hline Expectativas & "Supone un reto importante". \\
\hline Conclusiones & $\begin{array}{l}\text { "Sí con protocolo de "triage"”. } \\
\text { "Estamos muy preparadas para hacerlo. En un centro rural tuve una parada y pude con ello". } \\
\text { "Te das cuenta de que tenemos los conocimientos adecuados". } \\
\text { "Alto nivel de cualificación de enfermería". }\end{array}$ \\
\hline
\end{tabular}

Tabla 3. Opiniones del grupo focal de enfermeras sobre las diferentes propuestas de estrategias para el desarrollo de competencias en el proyecto sobre "triage" 


\begin{tabular}{l|l}
\hline Valoración inicial & $\begin{array}{l}\text { "Es algo desconocido y asusta. La gente es reacia a los cambios". } \\
\text { "Existe una sobrecarga, es así porque es así, nadie se ha planteado que esto puede cambiar, aunque } \\
\text { no me gusta que sea así". } \\
\text { "El hecho de darle una cita inmediata al paciente puede favorecer el aumento en la demanda". } \\
\text { "La accesibilidad aumenta la demanda". } \\
\text { "Más vale lo malo conocido que lo bueno por conocer". } \\
\text { "Problema de competencias y conflicto de intereses entre la profesión médica y la enfermería". } \\
\text { "Intentamos otorgar una función a la enfermería que la debería realizar un médico". } \\
\text { "A no ser que tengamos respaldo de la profesión médica no nos lo van a permitir". } \\
\text { "Tiene unas cualidades desarrolladas, estamos más capacitados que un administrativo, pero } \\
\text { necesitamos respaldo, información y formación". } \\
\text { "Sí que podemos decir un posible diagnóstico". } \\
\text { "Las técnicas, los cuidados, el trato con el paciente es más cómodo al inicio de terminar la carrera, } \\
\text { porque has rotado." }\end{array}$ \\
\hline Necesidades & $\begin{array}{l}\text { "No tenemos los conocimiento suficientes nada más salir de la Facultad" } \\
\text { "La experiencia no te la da la carrera". } \\
\text { "Se debe crear una especialidad de Enfermera de "triage" en atención primaria y Urgencias". }\end{array}$ \\
\hline Conclusiones & "Esta propuesta apuesta por el derecho a la intimidad del paciente". \\
\hline
\end{tabular}

Tabla 4. Opiniones del grupo focal de estudiantes sobre las diferentes propuestas de estrategias para el desarrollo de competencias en el proyecto sobre "triage"

gestores del sistema, conozcan el funcionamiento, confíen en este proceso de clasificación como beneficio al usuario y conlleve ciertas características inherentes a la enfermería de urgencias ${ }^{4,5}$.

Rancaño García et al. ${ }^{3}$ realizaron un estudio piloto sobre un modelo de "triage" implantado en varios centros de salud de Asturias, cuyo objetivo fundamental se basaba en la creación y validación de una herramienta enfocada para los Servicios de Urgencias de atención primaria. Como conclusión a su estudio establecen que los sistemas de "triage" orientados a la atención primaria, dada la incidencia de patología menos grave que en otros niveles de atención, deberían tener en cuenta esta circunstancia siendo más sensibles a patologías potencialmente importantes, aunque su gravedad real en el momento de atención sea menor. La herramienta de "triage" ("triage" en atención primaria: "TAP") ha conseguido uno de sus principales objetivos, que consiste en clasificar a los pacientes más "leves", diferenciándolos de aquellos en los que su patología es potencialmente más importante.

No hemos encontrado en la literatura ningún estudio similar al nuestro. Esto nos ha permitido conocer de primera mano los problemas reales y las dificultades en el abordaje asistencial que puede suponer la implantación de un protocolo específico mediante el análisis crítico sobre el liderazgo de un colectivo.
Asumimos que el proceso de selección de estos profesionales pude haber estado sesgado, al incluir profesionales especialmente motivados, con un gran interés en formar parte del proyecto. También por tratarse de profesionales con más de 10 años de experiencia en el ámbito de la atención primaria frente a estudiantes en proceso de formación, sin experiencia asistencial, aunque nos pareció que la de éstos era un punto de vista de gran interés.

Ante la pregunta sobre quién debe realizar el "triage", la gran mayoría de las opiniones recopiladas están en consonancia con la literatura médica. Así, el acuerdo general de enfermeras y estudiantes sobre la factibilidad en esta tarea ya ha sido descrito por Larrumbide et al. ${ }^{4}$, así como por Rao et al. ${ }^{6}$, donde ya afirmaban que la recepción y clasificación en Urgencias debe ser propia de enfermeras.

Sugirieron la posibilidad de mejorar la eficiencia del proyecto proponiendo a un profesional sanitario para resolver directamente las urgencias que pudiesen surgir, posibilidad no factible dada la situación de crisis actual, y las dificultades en la contratación de personal.

Si enfermeras con una larga trayectoria profesional coinciden en que es una tarea asumible y factible y proponen actividades para la recepción, acogida y clasificación, ¿por qué ha fracasado la implantación de esta propuesta? 
Hablan de desconocimiento, miedo al cambio, rutinas asumibles, necesidades formativas. En un estudio realizado por Orellana Yañez et al. ${ }^{7}$, ante el objetivo de proponer estrategias para desarrollar en los estudiantes una inquietud investigadora hacia una práctica basada en la evidencia, surge la necesidad de abordar primero el modelo de enfermería basada en la evidencia (EBE) y en segundo lugar las competencias de pensamiento crítico, reflexivo y habilidad de búsqueda de información, como bases que sustentan la investigación hacia la práctica de la EBE, así como la propuesta de algunas estrategias didácticas factibles de ser implementadas en las currícula de las unidades formadoras de profesionales de enfermería.

En cuanto a las dificultades para asumir la responsabilidad, que lo expresan como problemas de competencia entre diferentes profesionales sanitarios, Retsas ${ }^{8}$ identifica como las barreras más significativas para no usar la evidencia en orden de prioridad: el insuficiente tiempo de las enfermeras para implementar nuevas ideas en su trabajo, insuficiente tiempo para leer investigaciones, organizaciones que no entregan las estructuras necesarias para aplicar las evidencias, seguido por la dificultad que presentan las enfermeras para comprender las estadísticas, sensación de aislamiento de las enfermeras de sus colegas para discutir los hallazgos y la falta de colaboración de los médicos, entre otras.

En España, Martínez Riera ${ }^{9}$ concluye que la enfermería no está basada en las evidencias debido a la escasa investigación, siendo las principales barreras las de tipo organizacional por falta de orientación y conciencia de la importancia de la investigación, seguidas por un aislamiento dado por la escasa referencia y lectura de artículos en inglés, escasez de replicación, falta de financiación, dificultad en el acceso a fuentes y el aumento de la investigación cualitativa, de poco interés en las organizaciones que se guían por paradigmas positivistas. En cuanto a la opinión de los estudiantes sobre otras de las afirmaciones basadas en la reflexión de no asumir cambios en contraposición a una rutina, Gálvez ${ }^{10}$ apuntaba que existen barreras institucionales y organizativas, pero que tienen mayor valor las entregadas por la cultura profesional y la tendencia al inmovilismo de las enfermeras, dado por diferentes razones, entre las que nombra la falta de conocimiento, motivación insuficiente y negación a asumir responsabilidades.
Existe unanimidad en la afirmación referente a las necesidades formativas para asumir esta tarea que consideran de alta especialización. Martínez Riera ${ }^{9}$ identificó como los instrumentos más importantes para la implementación de la EBE: el soporte de los directivos; la adecuada cultura organizacional que cree, favorezca y potencie la EBE; la formación superior que permita el acceso a los máximos niveles de gestión, docencia e investigación; y la creación de equipos de investigación que lideren la práctica basada en evidencias.

Como conclusión a nuestro estudio, hemos de subrayar que enfermeras cualificadas en el trabajo en atención primaria y estudiantes de enfermería en la fase final de su formación coinciden en la factibilidad de implantar un sistema de "triage" para pacientes que requieren atención inmediata en un centro de salud, lo que supondría una mejora en la calidad y la eficacia de los servicios mediante una respuesta adecuada ante las necesidades de los pacientes, la gestión adecuada de la demanda y todo ello desde una perspectiva integradora humana y profesional.

Son más de 20 años de experiencia en "triage" en hospitales y son más de 30 años los que avalan a un colectivo de enfermeros de atención primaria caracterizado por sus aptitudes y habilidades comunicativas con el paciente y familiares, por su empatía y claridad de juicio clínico, en su capacidad de resolución ante situaciones conflictivas, capacidad en la atención integral del niño enfermo, del sano, del adulto y del anciano, en prevención...

Posiblemente debamos establecer un escenario propicio, unos protocolos bien definidos, un mayor respaldo médico e institucional para que estas competencias profesionales adquieran un papel relevante en una atención de excelencia.

\section{BIBLIOGRAFÍA}

1. Puigblanqué $E$, Noheras $M$, Nogueras $M$, Perapoch N. El triaje en el servicio de urgencias. Enferm Clin. 2008; 18: 3-4.

2. Manual para la implementación de un sistema de triaje para los cuartos de urgencias. Washington, DC.: OPS; 2011

3. Rancaño García I, Cobo Barquín JC, Cachero Fernández R, Hernández Mejía R. Triaje en Atención Primaria: sistema "TAP". E-notas de evaluación. Consejería de Sanidad del Principado de Asturias; 2011.

4. Larrumbide $\mathrm{H}$. La recepción y clasificación en urgencias debe ser propia de enfermeras. Enfermería de Actualidad 1999; 36:8. 
5. Sociedad Española de Enfermería de Urgencias (SEEU). De las funciones y tareas propias de los diplomados de enfermería en la recepción, acogida y clasificación de los pacientes en el área de urgencias. Recomendación científica 99/01/01 de 15 de junio de 1999 [URL disponible 1/12/2011: http://www. urgencias-medicas.org/colabora/jerotriage.pdf].

6. Rao GP, Hughes A. Nurse triage may reduce workload in accident department. Br Med J. 1995; 311: 1575.

7. Orellana Yáñez A, Paravic Klijn T. Enfermería Basada en Evidencia: Barreras y Estrategias para su implementación. Cienc Enferm. 2007; 13: 17-24.
8. Retsas A. Barriers to using research evidence in nursing practice. J Adv Nursing. 2000; 31: 599- 606.

9. Martínez Riera JR. Barreras e instrumentos facilitadores de la Enfermería Basada en la Evidencia. Enferm Clin. 2003; 13: 303-8.

10. Gálvez A. Evidencias, pruebas científicas y enfermería. Reflexión en voz baja y pensamientos inconfesables. Enferm Global. 2003; 2:1-9. 